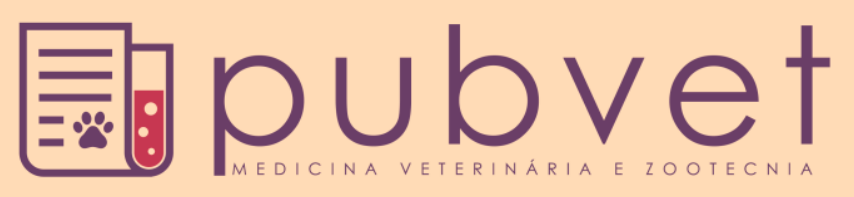

https://doi.org/10.22256/pubvet.v12n2a24.1-13

\title{
Técnicas de vídeo imagem para avaliação de carcaça: Revisão
}

\author{
André Torres Geraldo ${ }^{\ominus} 1^{*}$, Celia Raquel Quirino ${ }^{\ominus 1,2}$, Fabio da Costa Henry ${ }^{\ominus}$, Ricardo \\ Lopes Dias da $\operatorname{Costa}^{\ominus 4}$, Renato Travassos Beltrame ${ }^{\bullet}$
}

${ }^{1}$ Laboratório de Biotecnologia da Reprodução e Melhoramento Genético Animal, Universidade Estadual do Norte Fluminense, $R J$-Brasil. ${ }^{2}$ Pesquisadora do Conselho Nacional de Desenvolvimento Científico e Tecnológico - CNPq

${ }^{3}$ Laboratório de Zootecnia, Universidade Estadual do Norte Fluminense, RJ-Brasil

${ }^{4}$ Pesquisador do Instituto de Zootecnia, Centro de zootecnia diversificada, Nova Odessa, SP - Brasil

${ }^{5}$ Professor do Centro Universitário do Espírito Santo, Colatina, ES -Brasil

*Autor para correspondência: andre_atg@hotmail.com

\begin{abstract}
RESUMO. Várias técnicas são utilizadas para avaliar, tipificar e classificar carcaças de animais de interesse zootécnico, para fins de seleção de animais que apresentem as características desejadas, ou avaliar o produto gerado pelos animais destinados ao abate. Os métodos mais antigos e comumente utilizados são aqueles de aplicação mais prática e menos acurada, como as avaliações através do peso vivo ou escore corporal. No entanto, técnicas como a ultrassonografia, tomografia computadorizada, análise de vídeo imagem e scanning eletromagnético, vem sendo utilizadas para tornar as avaliações mais rápidas e acuradas, representando um progresso importante para produtores e para a indústria da carne.
\end{abstract}

Palavras chave: Scanning eletromagnético, tomografia computadorizada, ultrassonografia

\section{Video image technique for carcass evaluation: Review}

ABSTRACT. Several techniques have been used to evaluate, typify and classify carcasses of animals of zootechnical interest with the purpose of select animals with desirable traits or evaluate the products resulting from slaughtered animals. The older and most common methods that have been used are more practical and less accurate, such as body weight or body condition scoring. However, techniques such as ultrasonography, computed tomography, video image analysis and electromagnetic scanning enable faster and more accurate evaluations, representing an important progress for producers and for the meat industry.

Keywords: Computed tomography, electromagnetic scanning, ultrasonography

\section{Técnicas de video imagen para evaluación de canal: Revisión}

RESUMEN. Varias técnicas son utilizadas para evaluar, tipificar y clasificar canales de animales de interés zootécnico, para fines de selección de animales que presenten las características deseadas, o evaluar el producto generado por los animales destinados al sacrificio. Los métodos más antiguos y comúnmente utilizados son aquellos de aplicación más práctica y menos precisa, como las evaluaciones a través del peso vivo o escore corporal. Sin embargo, técnicas como la ultrasonografía, tomografía computarizada, análisis de vídeo imagen y escáner electromagnético, vienen siendo utilizadas para tornar las evaluaciones más rápidas y precisas, representando un progreso importante para productores y para la industria de la carne.

Palabras clave: Escaner electromagnético, tomografía computarizada, ultrasonografía 


\section{Introdução}

O conhecimento das diferenças entre as carcaças de animais destinados ao abate é uma importante ferramenta para a indústria animal, influenciando a destinação e o valor de mercado do produto. Carcaças de alto rendimento e que possuam as melhores caraterísticas são aquelas mais procuradas pelo mercado e que alcançam melhores ofertas (Brondani et al., 2006, Rotta et al., 2010).

O desenvolvimento de métodos não invasivos, rápidos e confiáveis para avaliação de carcaças tem sido um dos principais objetivos da indústria da carne (Dian et al., 2007, Dian et al., 2008). Em muitos países a técnica utilizada para tipificação e classificação das carcaças ainda é o método visual, pela pontuação para os atributos da carcaça, como cobertura de gordura subcutanêa e conformação muscular da carcaça (Osório et al., 2002) ou pelo peso que os animais apresentam por ocasião do abate, não se estabelecendo relação com as características de carcaça. Nesse caso, a seleção pelo modo visual se torna um estimador pouco preciso da composição da carcaça, tanto para os criadores, como para a indústria no momento da compra os animais (Tarouco et al., 2005). Esses métodos são subjetivos e trazem poucas informações sobre importantes caracteristicas. Várias técnicas existem para estimar a composição corporal, mas elas variam consideravelmente em custo, praticidade, rapidez e exatidão.

Com objetivo de tornar a avaliação de carcaças um processo mais eficiente e acurado várias tecnologias foram desenvolvidas, como a metodologia de análise pela tomografia computadorizada (Clelland et al., 2014), análise de vídeo imagem (Gupta et al., 2013), ultrassonografia (Orman et al., 2008) e por Scanning eletromagnético (TOBEC) (Higbie et al., 2002). No entanto, essas técnicas diferem em seus resultados e aplicações. Dessa forma, o objetivo dessa revisão é apresentar as principais tecnologias de vídeo imagem disponiveis para a avaliação de carcaça de animais de interesse zootécnico.

$\begin{aligned} & \text { Avaliação de carcaças: Tipificação } e \\ & \text { classificação }\end{aligned}$

Nos sistemas de avaliação de carcaças, o objetivo consiste primeiro classificar as mesmas, em classes, grupos, carcaças com características semelhantes (como sexo e maturidade). Já a etapa de tipificação consiste em ordenar as carcaças de modo hierárquico, de acordo com critérios de qualidade específicos, como: maturidade, peso, conformação, rendimento, gordura de cobertura, entre outras características (Sañudo \& Sierra, 1986).

A classificação de carcaças segundo a morfologia tem como objetivo estabelecer padrões para o mercado de carne, permitindo $o$ entendimento entre a oferta e a demanda, oferecendo ao consumidor diferentes categorias. Os principais motivos pelos quais diferentes países adotam o critério de conformação em seus sistemas de avaliação de carcaças devem-se, às expectativas de aumento nas porcentagens de cortes de alta qualidade, incremento na espessura dos músculos e melhor impressão que a carcaça bem conformada causa ao consumidor (Silva Sobrinho \& Silva, 2000).

O músculo é o principal componente quantitativo da carcaça, seguido da gordura e do osso, para bovinos, ovinos e suínos, e suas proporções dependem da raça. As curvas de crescimento dos tecidos muscular, ósseo e adiposo mostram que as quantidades de músculo e osso aumentam com a velocidade, proporcionalmente menores que o peso da carcaça. $\mathrm{O}$ peso de gordura aumenta mais rapidamente que o peso da carcaça, demonstrando a maturidade fisiológica de cada tecido, onde o ósseo é mais precoce, o muscular intermediário e o adiposo tardio (Silva Sobrinho \& Silva, 2000) (Figura 1).

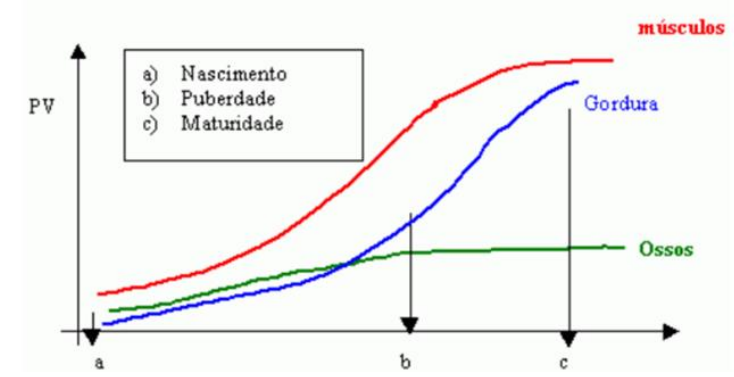

Figura 1. Curva de crescimento. Fonte: Owens et al. (1993).

A avaliação de carcaças pode ser conduzida pelas medidas objetivas e subjetivas, isoladas ou combinadas, por aspectos quantitativos e qualitativos como pesos, rendimentos, morfometrias e escores de conformação (Osório et al., 2002, Zundt et al., 2006).

\section{Sistemas de tipificação de carcaça bovina}

O Primeiro sistema de classificação de carcaças do mundo foi criado nos Estados Unidos 
em 1927, pelo Departamento de Agricultura dos Estados Unidos (USDA). Nesse sistema a classificação é dada pela classe de qualidade (Quality Grade) e pela classe de rendimento (Yeld Grade). Pela avaliação de qualidade as carcaças podem ser classificadas como: Prime, Choice, Selected e Standard para carcaças de animais mais jovens e Commercial, Utility e Cutter para carcaças de animais mais velhos (Felício, 2005). Nesse sistema as principais características avaliadas são a maturidade e o grau de marmoreio. Os graus de maturidade são: A (animal jovem), B, $\mathrm{C}, \mathrm{D}$ e $\mathrm{E}$ (animais velhos), com base no grau de ossificação da coluna vertebral. A determinação do marmoreio é realizada entre a $12^{\mathrm{a}}$ e $13^{\mathrm{a}}$ costelas, no músculo Longissimus dorsi, e classificado como abundante, moderadamente abundante, pouco abundante, moderado, modesto, pouco, leve, traços, e ausente (Müller, 1987). Pode-se dizer que há uma contradição entre o "Quality grade" e o "Yield grade", onde o primeiro classifica as melhores carcaças com o maior marmoreio, e a segunda desconta em função da maior quantidade de gordura. Geralmente as carcaças americanas são classificadas por um dos dois critérios, ou ambos (Strong, 2001).

O sistema de classificação de carcaças canadense foi criado tendo como base o americano. Desse modo, os dois sistemas apresentam semelhanças em seus critérios de classificação. Os principais critérios avaliados nesse sistema são maturidade, musculosidade, marmorização, cor da carne e espessura da gordura de cobertura. Animais jovens recebem classificação A ou B e carcaças de animais maduros são classificadas como D e de touros como E. As carcaças no Canadá, assim como nos EUA, também recebem classificação de acordo com o rendimento de tecido magro (Aalhus et al., 2004).

Na Europa o sistema utilizado baseia-se por escores de acabamento de carcaça e conformação de carcaça. Os escores são pontuados de 1 a 6 , segundo as letras S-E-U-R-O-P, sendo $\mathrm{P}$ as carcaças com menor pontuação e $\mathrm{S}$ as carcaças melhor pontuadas (Hickey et al., 2007).

O sistema japonês de avaliação de carcaça já está em um uso desde 1975 (sofreu modificações em 1988). Nesse sistema, ao contrário do americano, as avaliações de rendimento e qualidade são realizadas juntas. As notas para qualidade da carcaça são dadas por um escore de pontuação que vai de 1 (carcaça pobre) a 5 (carcaça excelente). As notas de rendimento de carcaça são como A, B e C. A nota final é feita combinando as duas, como B2, por exemplo. Ao contrário de outros países, as avaliações no Japão são realizadas no músculo Longissimus dorsi entre a $6^{\mathrm{a}}$ e $7^{\mathrm{a}}$ costelas (Strong, 2001).

O sistema de classificação e tipificação da Argentina classifica as carcaças em categorias de gênero, maturidade e peso, e tipifica pela conformação e gordura de cobertura. As de machos castrados são chamadas de terneros, que são carcaças muito leves, $100-146 \mathrm{~kg}$; novillitos, quando leves, pesando entre 148 e $236 \mathrm{~kg}$ e novillos, quando pesadas, mais de $236 \mathrm{~kg}$. As de fêmeas, chamadas de ternera, com 100-146 kg; vaquillona, entre 148 e $226 \mathrm{~kg}$ e vacas, pesando acima de $226 \mathrm{~kg}$. Por fim as de Toros, machos não castrados (ARGENTINA, 1973). Nesse sistema, os Novillos são tipificados quanto à conformação com as letras $\mathbf{J J}-\mathrm{J}-\mathrm{U}-\mathrm{U} 2-\mathrm{N}-\mathrm{T}-\mathrm{A}$, e todas as outras categorias de gênero-maturidade (Novillito, Ternero e Ternera, Vaquillona e Vaca) com as letras AA - A - B - C - D -E - F. Em ambas as sequências, as letras representam a conformação, da melhor para a pior, como exemplo, JJ ou AA = Superior; $\mathbf{J}$ ou $\mathrm{A}=$ Muy Buena; $\mathrm{U}$ ou $\mathrm{B}=$ Buena, e assim por diante (ARGENTINA, 1973).

O sistema de tipificação de carcaças australiano (Meat Standart of Australia - MAS (Polkinghorne \& Thompson, 2010)) é o mais novo e complexo sistema em uso, envolvendo diversos parâmetros para avaliação sendo introduzido no mercado em 1999. O MSA permite predizer, com relativa acurácia a qualidade de cada corte individual, de maneira simples e de fácil entendimento do consumidor. Ou seja, não mais a carcaça é classificada, mas sim cada corte, em função do modo de preparo. Para o correto funcionamento desse programa é necessário o controle e rastreamento dos animais durante a fase pré e pós abate (Polkinghorne \& Thompson, 2010).

O sistema brasileiro de tipificação é um esquema simples de classificação, seguido de hierarquização das carcaças em tipos. A classificação é feita quanto ao gênero e maturidade (estimada pela avaliação dos dentes incisivos permanentes) e a tipificação propriamente dita, pela combinação das classes de gênero e maturidade, com restrições de conformação, acabamento e peso de carcaça, para enquadramento nos tipos (Felício, 2005). A 
conformação é avaliada subjetivamente, dividindo as carcaças em cinco categorias: convexo; subconvexo; retilíneo; sub-retilíneo e côncavo. O acabamento também é avaliado de maneira subjetiva, estimando-se visualmente a quantidade de gordura na carcaça e dando escores de 1 a $5: 1$ $=$ ausência total de gordura; $2=1$ a $3 \mathrm{~mm} ; 3=3$ a $6 \mathrm{~mm} ; 4$ = 6 a $10 \mathrm{~mm} ; 5$ = acima de $10 \mathrm{~mm}$ de gordura de cobertura (Sainz \& Araujo, 2001).

\section{Peso e rendimento de carcaça}

O peso da carcaça é um dos fatores que apresenta maior influência na valorização do animal, havendo, em alguns países, preferências acentuadas e preços diferenciados segundo o peso da carcaça. Esse peso varia com o tipo de animal (genótipo) o sexo e a velocidade de ganho de peso (Rotta et al., 2009). O peso da carcaça é o primeiro critério considerado no momento da comercialização. Juntamente com fatores como a conformação, cobertura de gordura e cor da carne, expressa o conjunto de características utilizadas para definir uma carcaça de qualidade (Moreno et al., 2014). Em uma pesquisa realizada por Jucá et al. (2016) com ovinos Santa Inês, foram verificados pesos de carcaça quente e fria de 15,5 $\pm 4,18$ e $15,1 \pm 4,07 \mathrm{~kg}$, respectivamente. Outros estudos com a mesma raça citada, encontraram valores de 12,3 a 19,2 kg para o peso de carcaça quente como visto por Ribeiro \& Tedeschi (2012). Essas diferenças podem estar relacionadas com a idade de abate diante de acréscimos significativos e gradativos sobre parâmetros como, perda de água por cocção, perda de água por exsudação e força de cisalhamento nas distintas fases de criação dos animais (Guerrero et al., 2013b). Rosa et al. (2008) concluíram que o fator peso tem a capacidade de influenciar diretamente aspectos sensoriais do produto final. Além disso, fatores como o peso do animal vivo ou peso da carcaça, exercem uma forte influência sobre as características tecnológicas da carne, principalmente, sobre os níveis de gordura intramuscular (Missio et al., 2013).

Rendimento de carcaça é um termo usado comercialmente pelos matadouros e frigoríficos, e refere-se à diferença entre o peso vivo de animal e o peso da sua carcaça quente (obtido logo após o abate). O rendimento de carne magra depende da quantidade de músculos na carcaça e da sua relação com o conteúdo de ossos e gorduras (Gomide et al., 2009). O rendimento da carcaça bovina é uma medida diretamente relacionada ao aspecto econômico e, consequentemente, de maior interesse dos frigoríficos, que buscam maior rendimento para diluir os custos por quilograma de carne desossada (Pires, 2010, Guerrero et al., 2013a). O rendimento médio de carcaça do rebanho brasileiro é de 53\% (Rotta et al., 2009), e depende da quantidade de músculo em relação à ossatura e à gordura (Rotta et al., 2010).

McCurdy et al. (2010) constataram que o incremento no peso de abate melhora os rendimentos de carcaça quente e fria, quando expressos em relação ao peso de abate. Todavia, observaram similaridade de rendimentos, quando estes são expressos em relação ao peso de corpo vazio.

O peso total dos componentes externos, total de órgãos vitais, total de gordura interna e total do trato gastrintestinal vazio, em valores absolutos, aumenta com o incremento no peso de abate (Menezes et al., 2011). Todavia, quando ajustados para peso de abate e peso de corpo vazio, os pesos totais dos componentes externos e do trato gastrintestinal vazio apresentam similaridade, o total de órgãos vitais decresce e o total de gorduras internas se eleva com o aumento no peso de abate (Jerez-Timaure \& Huerta-Leidenz, 2009). No entanto, Vaz et al. (2013) avaliaram o rendimento de carcaça quente e de cortes comerciais em bovinos de diferentes pesos e idades e não constataram diferenças nos rendimentos.

\section{Técnicas de avaliação}

\section{Ultrassonografia em tempo real}

A ultrassonografia em tempo real (UTR) é uma importante ferramenta para avaliação in vivo da carcaça de animais destinados ao abate, podendo ser realizada momentos antes do abate para selecionar os animais que tenham as características de carcaças mais adequadas ao mercado alvo. A ultrassonografia em tempo real é umas das tecnologias que tem sido extensivamente estudada e utilizada para avaliação de características de carcaça de bovinos de corte (Valero et al., 2014). Resultados indicam que quando são obtidas por técnicos qualificados, as correlações entre as medidas por ultrassom e posteriormente na carcaça são altas (Greiner et al., 2003, Silva et al., 2003). A tecnologia também tem sido utilizada na prática como uma ferramenta para auxiliar os produtores na classificação de animais em grupos homogêneos para abate.

Fenômeno físico de ocorrência natural, o som, é um dos mais importantes eventos que fazem 
parte do conjunto da percepção sensitiva do ser humano, e pode ser definido como a propagação de uma vibração em um meio elástico (sólidos, líquidos e gasosos). As propagações de alta frequência ou ultrassom têm servido ao homem no âmbito das ciências médicas ocupando posição importante entre os mais eficazes métodos modernos de diagnósticos não invasivos. $\mathrm{O}$ desenvolvimento desta técnica se tornou importante na produção animal para a avaliação da composição e da qualidade da carcaça em animais vivos e têm mobilizado consideráveis recursos em pesquisas. Segundo Augusto \& Pachaly (2000), a qualidade da imagem gerada pelo ultrassom e sua correta interpretação dependem do conhecimento das interações entre as ondas e os tecidos ou órgãos que se deseja avaliar. $\mathrm{O}$ aparelho de ultrassom basicamente mede a reflexão das ondas de alta frequência que ocorrem quando estas passam através dos tecidos. Após o transdutor ter sido colocado em contato com o animal, o aparelho converte pulsos elétricos em ondas de alta frequência, que ao encontrar diferentes tecidos corpóreos dentro do animal promove uma reflexão parcial (eco) em tecidos menos densos, ou total em tecidos de alta densidade como os ossos (Yokoo et al., 2009). Mesmo após a ocorrência do eco, as ondas de alta frequência continuam a se propagar pelo corpo do animal e o conjunto de informações enviadas pelas reflexões transmitidas ao transdutor é projetado em uma tela como imagem onde as mensurações são realizadas (Augusto \& Pachaly, 2000).

A avaliação por ultrassonografia de carcaça em ovinos, caprinos e bovinos geralmente é realizada no músculo Longissimus dorsi, entre a $12^{\mathrm{a}}$ e $13^{\mathrm{a}}$ costelas, onde são tomadas medidas de altura do músculo, comprimento, área de olho de lombo e espessura de gordura subcutânea (Figura 2). Também existe a possibilidade da medição da gordura intramuscular (marmoreio), que pode ser realizada no mesmo corte, com auxílio de programas específicos ou de uma escala de pontuação. Inicialmente, deve-se realizar a tricotomia do local com apropriada contenção dos animais (Teixeira et al., 2008). A sonda deve ser posicionada sob uma pressão suficiente para que se assegure um bom contato acústico evitando a deformação dos tecidos e consequentemente, subestimar as medidas (Eiras et al., 2014).

Emenheiser et al. (2010) afirmam que as utilizações do equipamento por técnicos treinados podem predizer a qualidade da carne de cordeiros comerciais com grande precisão. Sugere ainda, estudos adicionais para avaliar as estimativas de espessura da gordura subcutânea como método de obter o rendimento de carcaça magra. Corroborando com Leeds et al. (2008) que encontraram correlação entre os valores obtidos pela ultrassonografia e os valores encontrados diretamente na carcaça de cordeiros em terminação. Orman et al. (2008) avaliando características de carcaça de cordeiros Awassi com diferentes pesos e sexos pela técnica de UTR, concluíram alta relação entre as avaliações in vivo e as carcaças de cordeiros, independente do sexo. Os autores ainda ressaltam que a utilização do peso corporal associado às medidas de ultrassom aumentou a confiabilidade da leitura. De outra forma, Ferreira et al. (2012) avaliando a correlação entre dados obtidos por UTR associada com um software de determinação digital de áreas à medidas de área de olho de lombo encontradas diretamente na carcaça, observaram altas correlações entre a técnica de imagem digital com as medidas na carcaça $(\mathrm{r}=0,89 ; 0,97$ e 0,98 , respectivamente), recomendando a utilização da UTR associada ao software devido à facilidade de execução, agilidade, e o baixo custo operacional.

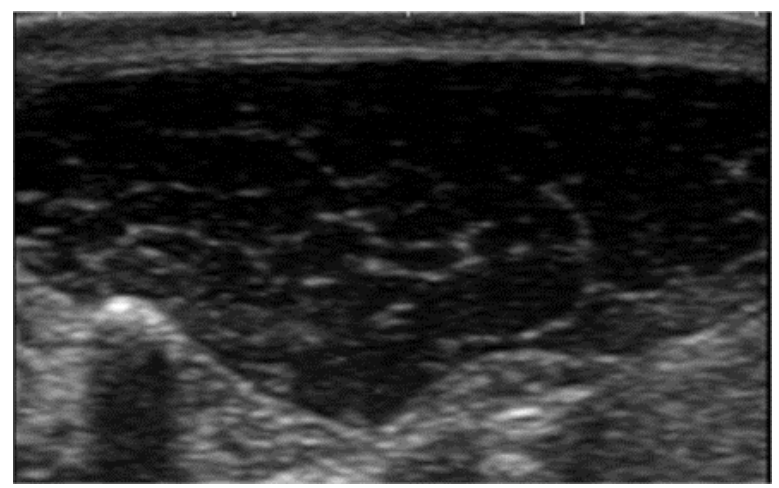

Figura 2. Imagem ultrassonográfica do músculo Longissimus dorsi. Fonte: Arquivo pessoal.

Lambe et al. (2010) encontraram alta correlação na quantidade e proporção de gordura subcutânea nos quartos dianteiro e traseiro $(\mathrm{r}=$ $0,80)$ com moderada precisão para o peso dos músculos $(\mathrm{r}=0,50$ a 0,60$)$ e baixos índices para as proporções musculares $(r=0,23$ a 0,49$)$ ao utilizar a técnica para avaliar a composição corporal in vivo pré-abate de bovinos jovens. Ressaltaram que realizando a UTR no final da terminação dos animais associada com o peso corporal possibilitou estimar a conformação e o estado de acabamento dos animais com moderada exatidão $(r=0,40$ e 0,6 , respectivamente). 


\section{Scanning eletromagnético (TOBEC)}

A varredura eletromagnética da carcaça, também conhecida como TOBEC (Total Body Electrical Conductivity), se baseia na diferença de condutividade elétrica que existe entre o tecido magro (muscular) e adiposo da carcaça (Gupta et al., 2013). Esta tecnologia sofre alguns problemas relacionados à temperatura, forma de apresentação, e custos de operação e manutenção. Entretanto, ela está sendo aplicada em várias indústrias de suínos nos EUA e no Canadá.

Os scanners eletromagnéticos consistem em uma bobina de fio de cobre enrolada em torno de um grande tubo de plexiglass, que forma a câmara de varredura (Stanford et al., 1998). Quando a corrente é aplicada à bobina, um campo eletromagnético de 2,5 MHz é criado. Carcaças ou cortes de carne passando pelo campo absorvem energia da bobina. A quantidade de absorção de energia detectada na bobina é um índice da massa condutora da carcaça ou cortes de carne. Uma vez que a massa sem gordura é aproximadamente 20 vezes mais condutora do que a gordura, o índice de condutividade está altamente correlacionado com a massa de tecido magro. Esta medição é obtida sem qualquer contacto elétrico direto com a amostra (Gupta et al., 2013). Higbie et al. (2002) avaliaram a exatidão do sistema TOBEC na predição do peso do tecido muscular e o peso da gordura em 32 carcaças de suínos, em comparação com a dissecação fisica. Eles relataram um valor $\mathrm{R}^{2}$ de 0,91 ao usar as medidas da varredura do lado esquerdo da carcaça na equação para prever o peso muscular.

Berg et al. (1997) avaliaram o sistema TOBEC e a impedância bioelétrica para sua precisão na previsão de carne magra dissecada total em 106 carcaças de cordeiro. As melhores equações TOBEC para prever os pesos de músculo e gordura em carcaças quentes obtiveram valores de $\mathrm{R}^{2}$ de 0,88 e 0,88 , respectivamente. Ambas as equações incluíam o peso da carcaça. As melhores equações para prever os valores de $R^{2}$ para as equações baseadas nas leituras TOBEC de carcaça refrigerada foram de 0,62 e 0,62 , respectivamente. Jones et al. (1996) realizaram um experimento para determinar as melhores equações de predição que usando o sistema TOBEC (Figura 3), no modelo para estimativa de gordura total livre, peso total de gordura, peso do tecido muscular e peso do pernil. A equação que mostrou melhores resultados foi aquela realizada em carcaças quentes $(\mathrm{Rq}=0,91)$. Os autores concluíram que o sistema TOBEC apresenta resultados excelentes na predição dos tecidos avaliados.

Berg et al. (1994) avaliaram a varredura eletromagnética de carcaças inteiras de suínos em uma configuração industrial on-line, integrada. $\mathrm{O}$ sistema TOBEC foi instalado em 2 plantas de processamento de carne suína, que processavam tanto carcaças quentes quanto frias. Os autores concluíram que o sistema TOBEC tem potencial para ser implantado em estrutura on line, apresentando ótimos resultados em configuração industrial totalmente on-line e automatizada.

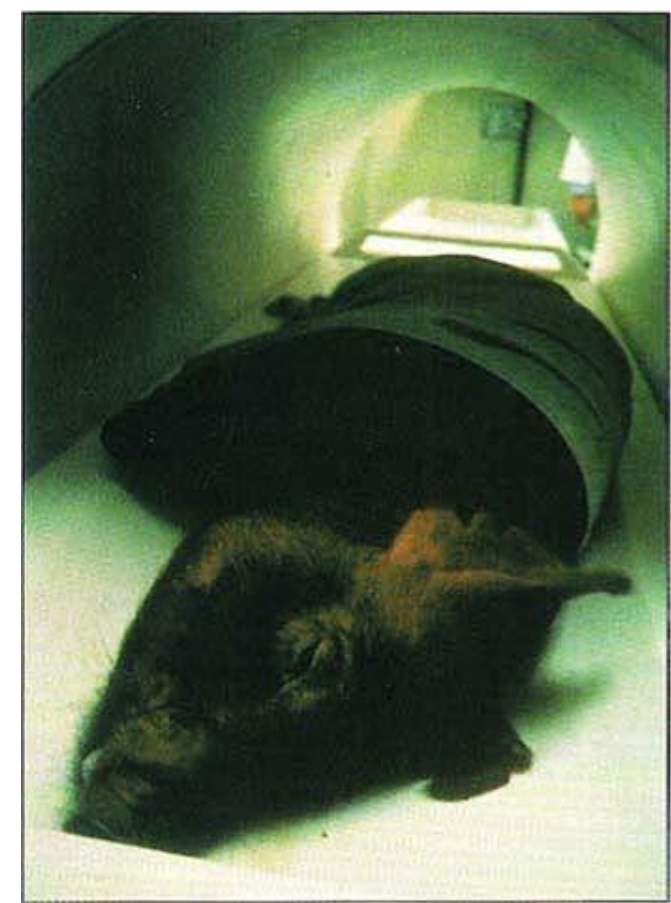

Figura 3. Exame de varredura eletromagnética em suínos.

\section{Análise de vídeo imagem}

Outra tecnologia já utilizada em diferentes áreas do conhecimento, e aplicada com sucesso na produção animal é a análise de vídeo imagem (AVI) (Figura 4). Por essa tecnologia é possível avaliar várias características quali e quantitativas da carcaça, como rendimento e qualidade de carne, de forma rápida sem interferências importantes na rotina industrial (Craigie et al., 2012, Craigie et al., 2013).

A tecnologia AVI funciona através da geração de um "mapa" elétrico, captado por câmera de vídeo e analisado através de softwares, que tem o poder de delimitar diferentes tecidos, como a gordura e tecido magro (Gupta et al., 2013). A VIA opera com base no princípio de que a intensidade de luz diferente recebida pelo elemento fotossensível da câmara de vídeo gera 
tensões diferentes de modo que áreas de luz (gordura) podem ser quantitativamente diferenciadas de áreas de áreas escuras (magra) (Stanford et al., 1998).

A pesquisa inicial sobre a avaliação de carcaças através de VIA foi realizada por Cross et al. (1983) que um potencial considerável de VIA como um dispositivo de classificação de rendimento para fins comerciais ou de pesquisa. Os cientistas descobriram que VIA tinha maior ou igual sucesso de previsão de massa magra muscular, quando comparado com um avaliador expert da USDA (United States Department of Agriculture).

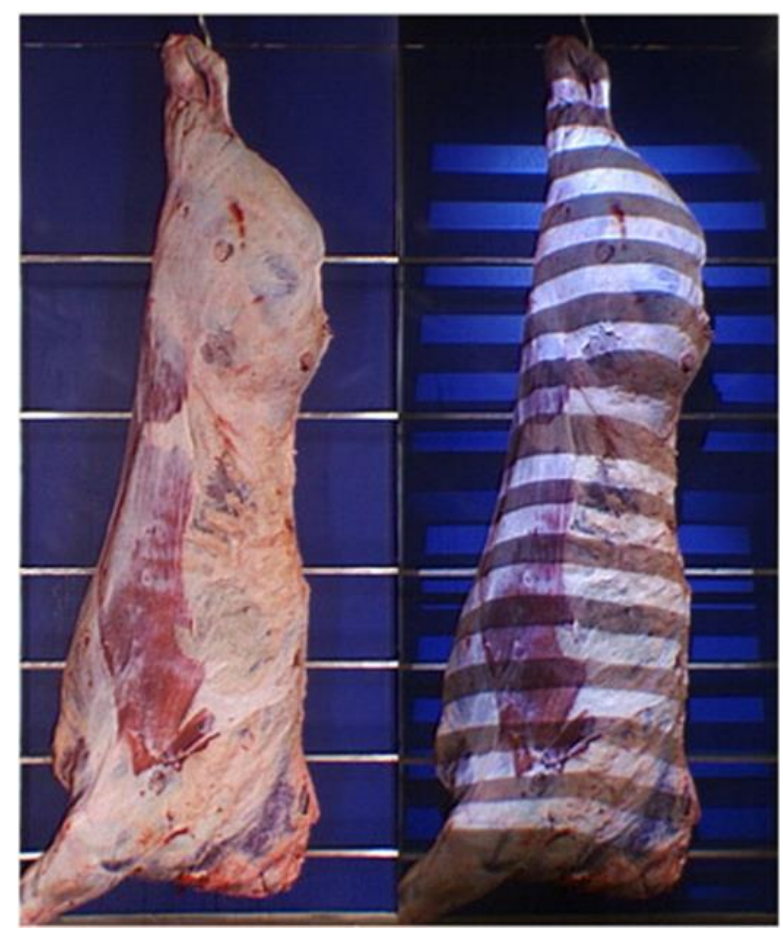

Figura 4. Análise de vídeo imagem VBS 2000. Fonte: E+V GmbH, Germany

Já são relatadas diversas pesquisas em que foram avaliados parâmetros como, maciez de carne, predição de gordura intramuscular, rendimento de carcaça e características de crescimento em diversas espécies de interesse zootécnico, como: suínos (Kashiha et al., 2014), bovinos (Pabiou et al., 2011, Eiras et al., 2016, Ornaghia et al., 2017, Rivaroli et al., 2017), ovinos (Einarsson et al., 2015), aves (Chmiel et al., 2011).

A VIA tem o potencial de ser aplicada na rotina da indústria animal por ser um processo que implica relativo baixo custo, não apresenta interação entre a carcaça e o avaliador (Carabús et al., 2016) e, acima de tudo, pela velocidade de avaliação, cerca de 1,5 no caso de suínos (Schofield, 2007). Por esses motivos a tecnologia vem senso usada com sucesso para classificar as carcaças em categorias de pagamento e para melhorar a consistência da classificação SEUROP em comparação com a avaliação visual (Craigie et al., 2012, Engel et al., 2012).

\section{Tomografia computadorizada (TC)}

A TC é uma tecnologia sofisticada, que pode oferecer informações objetivas e confiáveis. É uma técnica minimamente invasiva que pode ser usada para avaliação in vivo ou das carcaças após $\mathrm{o}$ abate, fornecendo estimativas altamente precisas da composição da carcaça (Quirino et al., 2016). A tecnologia é utilizada na clínica médica como forma de diagnóstico desde 1970; a partir de 1980 começou a ser utilizada na Europa como forma de predição da composição de carcaça e crescimento animal (Standal, 1984). No início, os trabalhos foram realizados com suínos, em seguida com ovinos, em carcaças e animais vivos (Sonesson et al., 1998, Jones et al., 1999), já sendo usada atualmente para classificar e tipificar as carcaças pelo sistema europeu de classificação de carcaças ovinas (EUROP) (Kongsro et al., 2008). Está análise é feita pelo uso de raios-x que geram imagens bidimensionais transversais do corpo, e cada imagem é obtida pela rotação de um tubo de raio-X de $360^{\circ}$ em torno do corpo do animal (Figura 5) (Clelland et al., 2014).

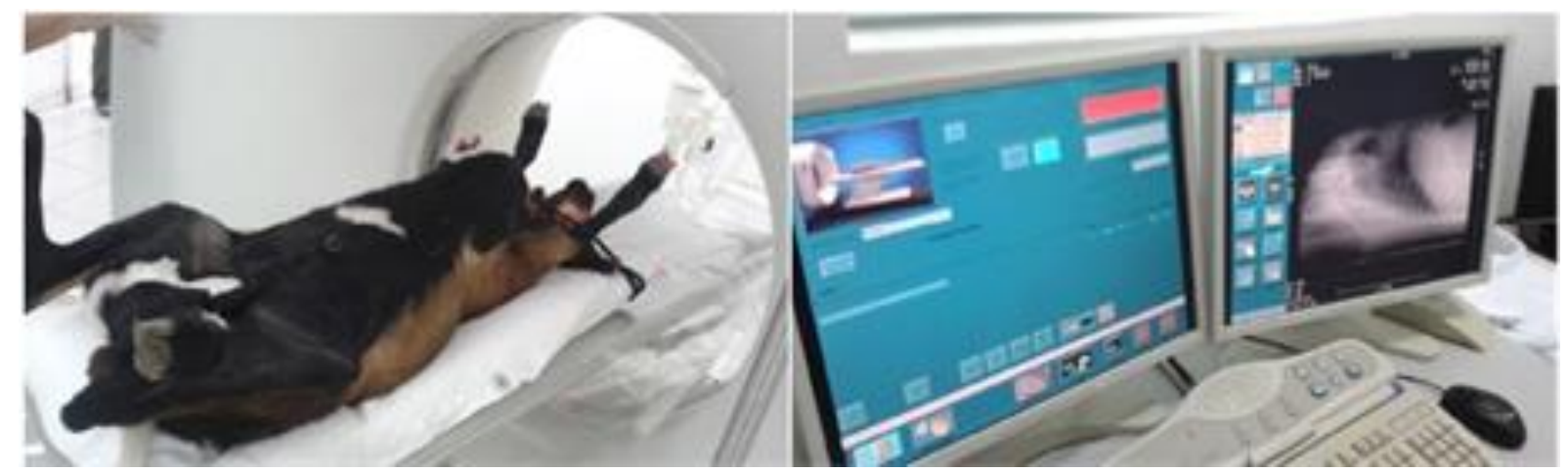

Figura 5. Analise tomografia em ovinos. Fonte: Geraldo et al. (2017). 
Após a digitalização da imagem, o objeto (corpo ou corte do animal) é dividido em várias seções consecutivas, paralelas e os dados são somados para produzir estimativas totais dos diferentes tecidos da carcaça. As imagens geradas são descritas em vários tons de cinza, criando uma imagem espacial do objeto digitalizado (Wegener, 1992).

A TC fornece previsões precisas de pesos de tecidos por causa de uma relação entre atenuação de raios $X$ e densidade de tecido. Os procedimentos de software para extrair e quantificar as áreas dos diferentes tecidos nas imagens transversais foram desenvolvidos usando algoritmos matemáticos para análise de imagem (Glasbey \& Young, 2002). O corte transversal em três locais identificados a partir da varredura topográfica $\left(5^{\mathrm{a}}\right.$ vértebra lombar (VL5)), $8^{\mathrm{a}}$ vértebra torácica (VT8) e ísquio (ISQ) (Figura 6) fornecem imagens adequadas para medições da área muscular, gordura e ossos, além de prever com boa precisão o peso dos componentes (Anderson et al., 2015). Este modo de análise foi desenvolvido com intenção de maximizar a precisão da leitura além de proceder à análise no menor tempo possível, para minimizar as implicações ao bem estar dos animais (Bünger et al., 2011). Outro método utilizado é realizado pela varredura completa do corpo do animal, denominado método Cavalieri, no entanto esse método é mais demorado (Navajas et al., 2007).

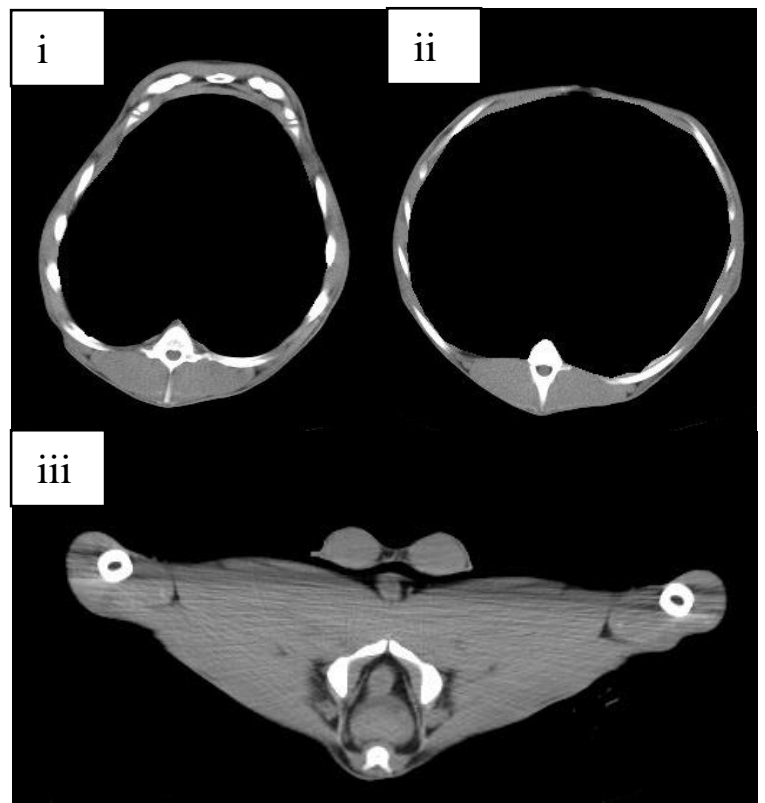

Figura 6. Topogramas e imagens transversais da oitava vértebra torácica (i) quinta vértebra lombar (ii) e Ísquio (iii) Fonte: Arquivo Pessoal.
O Uso do TC como para pesquisas em produção animal iniciou-se na década de 1980 na Escócia, sendo que seus resultados indicaram considerável potencial para predição de carcaça em animais vivos (Allen \& Leymaster, 1985). Devido a fatores como pouco acesso ao equipamento, e alto custo de execução, a TC não foi uma tecnologia amplamente difundida para predição de carcaças (Rivero et al., 2005).

Vários estudos foram realizados para avaliar a carcaça e predizer a composição corporal de ovinos e suínos tendo a dissecação manual como referência (Szabo et al., 1999, Jones et al., 2002, Lambe et al., 2006, Dobrowolski et al., 2004, Johansen et al., 2007, Navajas et al., 2007, Panea et al., 2012).

Os resultados demonstraram boas correlações entre as proporções dos tecidos de carcaça, estimadas por tomografia computadorizada, quando compradas a dissecação realizada por técnicos treinados (Sehested, 1986), com menor erro padrão com relação as estimativas realizadas por ultrassonografia, técnica amplamente utilizada na predição de tecido de carcaça até hoje (Suguisawa, 2002, Suguisawa \& Soares, 2006). A alta correlação entre os valores obtidos pela tomografia computadorizada e da dissecação manual em carcaça pode tornar a dissecação obsoleta para o futuro, visto que a tomografia representa um método mais rápido, e extremamente acurado na predição de tecidos de carcaça (Johansen et al., 2007).

\section{Conclusão}

A avaliação e tipificação de carcaças de animais de interesse zootécnico através de métodos inovadores e tecnológicos é uma ferramenta que garante resultados mais confiáveis e menos invasivos sobre as reais características de crescimento e deposição tecidual. No entanto, cada tecnologia possui vantagens e desvantagens, como custo de implementação e tempo necessário para análise, desse modo à escolha da técnica utilizada deve ser feita com critério, de modo a se adequar as necessidades da indústria.

\section{Referências Bibliográficas}

Aalhus, J. L., Dugan, M. E. R., Robertson, W. M., Best, D. R. \& Larsen, I. L. 2004. A withinanimal examination of postmortem ageing for up to $21 \mathrm{~d}$ on tenderness in the bovine longissimus thoracis and semimembranosus 
muscles. Canadian Journal of Animal Science, 84, 301-304.

Allen, P. \& Leymaster, K. A. 1985. Machine error in X-ray computer tomography and its relevance to prediction of in vivo body composition. Livestock Production Science, 13, 383-398.

Anderson, F., Williams, A., Pannier, L., Pethick, D. W. \& Gardner, G. E. 2015. Sire carcass breeding values affect body composition in lambs-1. Effects on lean weight and its distribution within the carcass as measured by computed tomography. Meat science, 108, 145-154.

ARGENTINA. 1973. Clasificación y Tipificación Oficial de la Carnes Vacunas. In: Nácion, M. d. A. y. G. d. 1. (ed.). Junta Nacional de Carnes., Buenos Aires.

Augusto, A. Q. \& Pachaly, J. R. 2000. Princípios físicos da ultra-sonografia-Revisão bibliográfica. Arquivos de Ciências Veterinárias e Zoologia da UNIPAR, 3, 61-65.

Berg, E. P., Forrest, J. C. \& Fisher, J. E. 1994. Electromagnetic scanning of pork carcasses in an on-line industrial configuration. Journal of Animal Science, 72, 2642-2652.

Berg, E. P., Neary, M. K., Forrest, J. C., Thomas, D. L. \& Kauffman, R. G. 1997. Evaluation of electronic technology to assess lamb carcass composition. Journal of Animal Science, 75, 2433-2444.

Brondani, I. L., Sampaio, A. A. M., Restle, J., Alves Filho, D. C., Freitas, L. D. S., Amaral, G. A., Silveira, M. F. \& Cezimbra, I. M. 2006. Composição física da carcaça e aspectos qualitativos da carne de bovinos de diferentes raças alimentados com diferentes níveis de energia. Revista Brasileira de Zootecnia, 35, 2034-2042.

Bünger, L., Glasbey, C. A., Simm, G., Conington, J., Macfarlane, J. M., McLean, K. A., Moore, K. \& Lambe, N. R. 2011. Use of X-ray computed tomography (CT) in UK sheep production and breeding. CT ScanningTechniques Applied, 1, 329-348.

Carabús, A., Gispert, M. \& Font-i-Furnols, M. 2016. Imaging technologies to study the composition of live pigs: A review. Spanish Journal of Agricultural Research, 14, 1-16.

Chmiel, M., Słowiński, M. \& Dasiewicz, K. 2011. Application of computer vision systems for estimation of fat content in poultry meat. Food Control, 22, 1424-1427.

Clelland, N., Bunger, L., McLean, K. A., Conington, J., Maltin, C., Knott, S. \& Lambe, N. R. 2014. Prediction of intramuscular fat levels in Texel lamb loins using X-ray computed tomography scanning. Meat Science, 98, 263-271.

Craigie, C. R., Navajas, E. A., Purchas, R. W., Maltin, C. A., Bünger, L., Hoskin, S. O., Ross, D. W., Morris, S. T. \& Roehe, R. 2012. A review of the development and use of video image analysis (VIA) for beef carcass evaluation as an alternative to the current EUROP system and other subjective systems. Meat Science, 92, 307-318.

Craigie, C. R., Ross, D. W., Maltin, C. A., Purchas, R. W., Bünger, L., Roehe, R. \& Morris, S. T. 2013. The relationship between video image analysis (VIA), visual classification, and saleable meat yield of sirloin and fillet cuts of beef carcasses differing in breed and gender. Livestock Science, 158, 169178.

Cross, H. R., Gilliland, D. A., Durland, P. R. \& Seideman, S. 1983. Beef carcass evaluation by use of a video image analysis system. Journal of Animal Science, 57, 908-917.

Dian, P. H. M., Andueza, D., Jestin, M., Prado, I. N. \& Prache, S. 2008. Comparison of visible and near infrared reflectance spectroscopy to discriminate between pasture-fed and concentrate-fed lamb carcasses. Meat Science, 80, 1157-1164.

Dian, P. H. M., Chauveau-Duriot, B., Prado, I. N. \& Prache, S. 2007. A dose-response study relating the concentration of carotenoid pigments in blood and reflectance spectrum characteristics of fat to carotenoid intake level in sheep. Journal of Animal Science, 85, 30543061 .

Dobrowolski, A., Branscheid, W., Romvari, R., Horn, P. \& Allen, P. 2004. X-ray computed tomography as possible reference for the pig carcass evaluation. Fleischwirtschaft, 84, 109112.

Einarsson, E., Eythorsdottir, E., Smith, C. R. \& Jonmundsson, J. V. 2015. Genetic parameters for lamb carcass traits assessed by video image analysis, EUROP classification and in vivo measurements. Icelandic Agricultural Sciences, 28, 3-14. 
Eiras, C. E., Araújo Marques, J., Novais, D. L., Mottin, C., Maggioni, D. \& Prado, I. N. 2014. Ultrassonografia na avaliação da composição corporal de ruminantes: Revisão. Campo Digital, 9, 110-116.

Eiras, C. E., Ornaghi, M. G., Valero, M. V., Rivaroli, D. C., Guerrero, A. \& Prado, I. N. 2016. How does the dietary cottonseed hull affect the carcass characteristics and meat quality of young bulls finished in a highconcentrate diet? Acta Scientiarum. Animal Sciences, 38, 301-310.

Emenheiser, J. C., Greiner, S. P., Lewis, R. M. \& Notter, D. R. 2010. Validation of live animal ultrasonic measurements of body composition in market lambs. Journal of Animal Science, 88, 2932-2939.

Engel, B., Lambooij, E., Buist, W. G. \& Vereijken, P. 2012. Lean meat prediction with HGP, CGM and CSB-Image-Meater, with prediction accuracy evaluated for different proportions of gilts, boars and castrated boars in the pig population. Meat Science, 90, 338344.

Felício, P. E. 2005. Classificação e tipificação de carcaças bovinas. I Congresso Brasileiro de Ciencia e Tecnologia de Carne. São Pedro, São Paulo.

Ferreira, O. G. L., Rossi, F. D., Coelho, R. A. T., Fucilini, V. F. \& Benedetti, M. 2012. Measurement of rib-eye area by the method of digital images. Revista Brasileira de Zootecnia, 41, 811-814.

Geraldo, A. T., Quirino, C. R., Beltrame, R. T. \& Costa, R. L. D. 2017. Avaliação de carcaça ovina por tomografia computadorizada: Estado da Arte no Brasil e no mundo. PUBVET: Publicações em Medicina Veterinária $e$ Zootecnia, 11, 91-102.

Glasbey, C. A. \& Young, M. J. 2002. Maximum a posteriori estimation of image boundaries by dynamic programming. Journal of the Royal Statistical Society: Series C, 51, 209-221.

Gomide, L. A. M., Ramos, E. M. \& Fontes, P. R. 2009. Tecnologia de abate e tipificação de carcaças. Viçosa: Editora UFV, 370 p. Editora UFV, Viçosa.

Greiner, S. P., Rouse, G. H., Wilson, D. E., Cundiff, L. V. \& Wheeler, T. L. 2003. The relationship between ultrasound measurements and carcass fat thickness and longissimus muscle area in beef cattle. Journal of Animal Science, 81, 676-682.
Guerrero, A., Prado, I. N. \& Valero, M. V. 2013a. Calidad de la carne de bovino de animales procedentes de sistemas intensivos, semi intensivos y pastoreo. In: Londrina, U. E. d. (ed.) Simpósio de Produção Animal a Pasto. Londrina, Paraná.

Guerrero, A., Valero, M. V., Campo, M. M. \& Sañudo, C. 2013b. Some factors that affect ruminant meat quality: from the farm to the fork. Review. Acta Scientiarum. Animal Sciences, 35, 335-347.

Gupta, S., Kumar, A., Kumar, S., Bhat, Z. F., Hakeem, H. R. \& Abrol, A. P. S. 2013. Recent trends in carcass evaluation techniques-a review. Journal of Meat Science and Technology, 1, 50-55.

Hickey, J. M., Keane, M. G., Kenny, D. A., Cromie, A. R. \& Veerkamp, R. F. 2007. Genetic parameters for EUROP carcass traits within different groups of cattle in Ireland. Journal of Animal Science, 85, 314-321.

Higbie, A. D., Bidner, T. D., Matthews, J. O., Southern, L. L., Page, T. G., Persica, M. A., Sanders, M. B. \& Monlezun, C. J. 2002. Prediction of swine carcass composition by total body electrical conductivity (TOBEC). Journal of Animal Science, 80, 113-122.

Jerez-Timaure, N. \& Huerta-Leidenz, N. 2009. Effects of breed type and supplementation during grazing on carcass traits and meat quality of bulls fattened on improved savannah. Livestock Science, 121, 219-226.

Johansen, J., Egelandsdal, B., Røe, M., Kvaal, K. \& Aastveit, A. H. 2007. Calibration models for lamb carcass composition analysis using Computerized Tomography (CT) imaging. Chemometrics and Intelligent Laboratory Systems, 87, 303-311.

Jones, H. E., Lewis, R. M., Young, M. J. \& Wolf, B. T. 2002. The use of X-ray computer tomography for measuring the muscularity of live sheep. Animal Science, 75, 387-399.

Jones, H. E., Simm, G., Dingwall, W. S. \& Lewis, R. M. 1999. Genetic relationships between visual and objective measures of carcass composition in crossbred lambs. Animal Science, 69, 553-561.

Jones, S. D. M., Robertson, W. M., Price, M. A. \& Coupland, T. 1996. The prediction of saleable meat yield in lamb carcasses. Canadian Journal of Animal Science, 76, 49-53. 
Jucá, A. F., Faveri, J. C., Melo Filho, G. M., Ribeiro Filho, A. L., Azevedo, H. C., Muniz, E. N., Pedrosa, V. B. \& Pinto, L. F. B. 2016. Effects of birth type and family on the variation of carcass and meat traits in Santa Ines sheep. Tropical Animal Health and Production, 48, 435-443.

Kashiha, M., Bahr, C., Ott, S., Moons, C. P. H., Niewold, T. A., Ödberg, F. O. \& Berckmans, D. 2014. Automatic weight estimation of individual pigs using image analysis. Computers and Electronics in Agriculture, 107, 38-44.

Kongsro, J., Røe, M., Aastveit, A. H., Kvaal, K. \& Egelandsdal, B. 2008. Virtual dissection of lamb carcasses using computer tomography (CT) and its correlation to manual dissection. Journal of Food Engineering, 88, 86-93.

Lambe, N. R., Conington, J., McLean, K. A., Navajas, E. A., Fisher, A. V. \& Bünger, L. 2006. In vivo prediction of internal fat weight in Scottish Blackface lambs, using computer tomography. Journal of Animal Breeding and Genetics, 123, 105-113.

Lambe, N. R., Ross, D. W., Navajas, E. A., Hyslop, J. J., Prieto, N., Craigie, C., Bünger, L., Simm, G. \& Roehe, R. 2010. The prediction of carcass composition and tissue distribution in beef cattle using ultrasound scanning at the start and/or end of the finishing period. Livestock Science, 131, 193-202.

Leeds, T. D., Mousel, M. R., Notter, D. R., Zerby, H. N., Moffet, C. A. \& Lewis, G. S. 2008. Bmode, real-time ultrasound for estimating carcass measures in live sheep: Accuracy of ultrasound measures and their relationships with carcass yield and value. Journal of Animal Science, 86, 3203-3214.

McCurdy, M. P., Horn, G. W., Wagner, J. J., Lancaster, P. A. \& Krehbiel, C. R. 2010. Effects of winter growing programs on subsequent feedlot performance, carcass characteristics, body composition, and energy requirements of beef steers. Journal of Animal Science, 88, 1564-1576.

Menezes, L. F. G., Brondani, I. L., Restle, J., Filho, D. C. A., Callegaro, A. M. \& Weise, M. 2011. Características dos componentes não integrantes da carcaça de novilhos superjovens da raça Devon, terminados em diferentes sistemas de alimentação. Arquivo Brasileiro de Medicina Veterinária e zootecnia, 63, 372381.
Missio, R. L., Restle, J., Moletta, J. L., Kuss, F., Neiva, J. N. M., Miotto, F. R. C., Prado, I. N., Elejalde, D. A. G. \& Perotto, D. 2013. Empty body components of cows of Purunã breed slaughtered at different weigths. Semina: Ciências Agrárias, 34, 883-894.

Moreno, G. M. B., Borba, H., Araújo, G. G. L., Voltolini, T. V., Souza, R. A., Silva Sobrinho, A. G., Buzanskas, M. E., Lima Júnior, D. M. \& Alvarenga, T. I. R. C. 2014. Rendimentos de carcaça, cortes comerciais e não-componentes da carcaça de cordeiros Santa Inês alimentados com feno de erva-sal e concentrado. Revista Brasileira de Saúde e Produção Animal, 15, 192-205.

Müller, L. 1987. Normas para avaliação de carcaça e concurso de carcaça de novilhos. Universidade Federal de Santa Maria, Santa Maria, Rio Grande do Sul, Brasil.

Navajas, E. A., Lambe, N. R., McLean, K. A., Glasbey, C. A., Fisher, A. V., Charteris, A. J. L., Bünger, L. \& Simm, G. 2007. Accuracy of in vivo muscularity indices measured by computed tomography and their association with carcass quality in lambs. Meat Science, 75, 533-542.

Orman, A., Çalışkan, G. Ü., Dikmen, S., Üstüner, H., Ogan, M. M. \& Çalışkan, Ç. 2008. The assessment of carcass composition of Awassi male lambs by real-time ultrasound at two different live weights. Meat Science, 80, 10311036.

Ornaghia, M. G., Passetti, R. A. C., Torrecilhas, J. A., Mottin, C., Vital, A. C. P., Gurerrero, A., Sañudo, C. \& Campo, M. M. 2017. Essential oils in the diet of young bulls: Effect on animal performance, digestibility, temperament, feeding behaviour and carcass characteristics. Animal Feed Science and Technology, 234, 274-283.

Osório, J., Osório, M., Oliveira, N. \& Siewerdt, L. 2002. Qualidade, morfologia e avaliação de carcaças, Pelotas.

Owens, F. N., Dubeski, P. \& Hanson, C. F. 1993. Factors that alter the growth and development of ruminants. Journal of Animal Science, 71, 3138-3150.

Pabiou, T., Fikse, W. F., Cromie, A. R., Keane, M. G., Näsholm, A. \& Berry, D. P. 2011. Use of digital images to predict carcass cut yields in cattle. Livestock Science, 137, 130-140.

Panea, B. D., Ripoll, G. G., Albertí, P. L., Joy, M. T. \& Teixeira, A. 2012. Atlas de disección de 
la canal de los rumiantes. Información Técnica Económica Agraria, 108, 5-105.

Pires, A. V. 2010. Bovinocultura de Corte. FEALQ, Piracicaba, São Paulo.

Polkinghorne, R. J. \& Thompson, J. M. 2010. Meat standards and grading: A world view. Meat Science, 86, 227-235.

Quirino, C. R., Geraldo, A. T., Antunes, F., Vieira, G. S., Rua, M. A. S., Freitas, A. C. B., David, C. M. \& Bartholazzi Júnior, A. 2016. Avaliação de carcaça de ovinos através da tomografia computadorizada. In: ALPA (ed.) XXV Congresso de la Associación Latinoamericana de Produción Animal. ALPA, Recife.

Ribeiro, F. R. B. \& Tedeschi, L. O. 2012. Using real-time ultrasound and carcass measurements to estimate total internal fat in beef cattle over different breed types and managements. Journal of Animal Science, 90, 3259-3265.

Rivaroli, D. C., Ornaghi, M. G., Mottin, C., Prado, R. M., Ramos, T. R., Guerrero, A., Jorge, A. M. \& Prado, I. N. 2017. Essential oils in the diet of crossbred ( $1 / 2$ Angus vs. $1 / 2$ Nellore) bulls finished in feedlot on animal performance, feed efficiency and carcass characteristics. Journal of Agricultural Science, 9, 205-212.

Rivero, M. A., Ramirez, J. A., Vazquez, J. M., Gil, F., Ramirez, G. \& Arencibia, A. 2005. Normal anatomical imaging of the thorax in three dogs: computed tomography and macroscopic cross sections with vascular injection. Anatomia, Histologia, Embryologia, 34, 215-219.

Rosa, G. T., Pires, C. C., Silva, J. H. S., Motta, O. S. \& Colomé, L. M. 2008. Composição tecidual da carcaça e de seus cortes e crescimento alométrico do osso, músculo e gordura da carcaça de cordeiros da raça texel. Acta Scientiarum. Animal Sciences, 24, 11071111.

Rotta, P. P., Prado, I. N. \& Prado, R. M. 2010. Desempenho, qualidade da carcaça e da carne em bovinos. In: Prado, I. N. (ed.) Produção de bovinos de corte e qualidade da carne. Eduem, Maringá, Paraná, Brasil.

Rotta, P. P., Prado, R. M., Prado, I. N., Valero, M. V., Visentainer, J. V. \& Silva, R. R. 2009. The effects of genetic groups, nutrition, finishing systems and gender of Brazilian cattle on carcass characteristics and beef composition and appearance: a review. Asian-Australasian Journal of Animal Sciences, 22, 1718-1734.
Sainz, R. D. \& Araujo, F. R. C. 2001. Tipificação de carcaças de bovinos e suínos. Congresso Brasileiro de Ciencia e Tecnologia de Carne. Campinas, Sã Paulo.

Sañudo, C. \& Sierra, A. I. 1986. Calidad de la canal en la especie ovina. Ovino. One.

Schofield, C. P. 2007. Portable image-based pig weight monitoring systems. Precision Livestock Farming '07. Wageningen Academic Publishers, Wageningen.

Sehested, E. 1986. In-vivo prediction of lamb carcass composition by computerized tomography. Department of Animal Science. Agricultural University of Norway.

Silva, S. L., Leme, P. R., Pereira, A. S. C. \& Putrino, S. M. 2003. Correlações entre características de carcaça avaliadas por ultrasom e pós-abate em novilhos Nelore, alimentados com altas proporções de concentrado. Revista Brasileira de Zootecnia, 32, 1236-1242.

Silva Sobrinho, A. \& Silva, A. 2000. Produção de carne ovina. Revista Nacional da carne, 24, 3244.

Sonesson, A. K., De Greef, K. H. \& Meuwissen, T. H. E. 1998. Genetic parameters and trends of meat quality, carcass composition and performance traits in two selected lines of Large White pigs. Livestock Production Science, 57, 23-32.

Standal, N. 1984. Establishment of CT facility for farm animals. In vivo measurement of body composition in meat animals. Elsevier Applied Science Publishers, London, 1, 43-51.

Stanford, K., Jones, S. D. M. \& Price, M. A. 1998. Methods of predicting lamb carcass composition: A review. Small Ruminant Research, 29, 241-254.

Strong, J. 2001. Carcass Grading SchemesUSA/Japan/Australia. How and Why Do They Differ? Proceedings of the Marbling Symposium.

Suguisawa, L. 2002. Ultra-sonografia para predição das características e composição da carcaça de bovinos. Universidade de São Paulo.

Suguisawa, L. \& Soares, W. R. M. 2006. Ultrasonografia para predição da composição da carcaça de bovinos jovens. Revista Brasileira de Zootecnia, 35, 177-185.

Szabo, C. S., Babinszky, L., Verstegen, M. W. A., Vangen, O., Jansman, A. J. M. \& Kanis, E. 
1999. The application of digital imaging techniques in the in vivo estimation of the body composition of pigs: a review. Livestock Production Science, 60, 1-11.

Tarouco, J. U., Lobato, J. F. P., Tarouco, A. K. \& Massia, G. S. 2005. Relação entre medidas ultra-sônicas e espessura de gordura subcutânea ou área de olho de lombo na carcaça em bovinos de corte. Revista Brasileira de Zootecnia, 34, 2074-2084.

Teixeira, A., Joy, M. \& Delfa, R. 2008. In vivo estimation of goat carcass composition and body fat partition by real-time ultrasonography. Journal of Animal Science, 86, 2369-2376.

Valero, M. V., Prado, R. M., Zawadzki, F., Eiras, C. E., Madrona, G. S. \& Prado, I. N. 2014. Propolis and essential oils additives in the diets improved animal performance and feed efficiency of bulls finished in feedlot. Acta Scientiarum. Animal Sciences, 36, 419-426.

Vaz, F. N., Restle, J., Pádua, J. T., Fonseca, C. A. \& Pacheco, P. S. 2013. Características de carcaça e receita industrial com cortes primários da carcaça de machos nelore abatidos com diferentes pesos. Ciência Animal Brasileira, 14, 199-207.
Wegener, O. H. 1992. Whole-body computed tomography. Blackwell Scientific Publications, Cambridge.

Yokoo, M. J. I., Werneck, J. N., Pereira, M. C., Albuquerque, L., Koury Filho, W., Sainz, R. D., Lobo, R. B. \& Araújo, F. 2009. Correlações genéticas entre escores visuais e características de carcaça medidas por ultrassom em bovinos de corte. Pesquisa Agropecuária Brasileira, 44, 197-202.

Zundt, M., Macedo, F. A. F., Astolphi, J. L., Mexia, A. A. \& Sakaguti, E. S. 2006. Desempenho e características de carcaça de cordeiros Santa Inês confinados, filhos de ovelhas submetidas à suplementação alimentar durante a gestação. Revista Brasileira de Zootecnia, 35, 928-935.

\section{Article History:}

Received 21 September 2017

Accepted 24 October 2017

Available online 3 Janeiro 2018

License information: This is an open-access article distributed under the terms of the Creative Commons Attribution License 4.0, which permits unrestricted use, distribution, and reproduction in any medium, provided the original work is properly cited. 